\title{
Prediction of Early Liver Failure in Pediatric Patients Admitted to Intensive Care Unit
}

\author{
Mozhgan Zahmatkeshan ${ }^{1}$, Zahra Serati ${ }^{2}$, Shole Freydooni ${ }^{2}$, Ali Reza Safarpour ${ }^{1, *}$, \\ Atefeh Esmailnejad ${ }^{3}$, Saeede Haghbin ${ }^{2}$
}

1. Gastrenterohepatology Research Center, Shiraz University of Medical Sciences, Shiraz, Iran

2. Department of Pediatrics, Shiraz University of Medical Sciences, Shiraz, Iran

3. Department of Pathobiology, School of Veterinary Medicine, Shiraz University, Shiraz, Iran

\footnotetext{
* Corresponding Author:
}

Ali Reza Safarpour, MD, PhD

Gastroenterohepatology Research Center, Shiraz University of Medical Sciences, Shiraz, Iran

Tel: +987112357282

Fax: + 987112307594

Email: Asafarpour@sums.ac.ir

Received: 18 Feb. 2018

Accepted: 10 Jun. 2019

\section{ABSTRACT}

\section{BACKGROUND}

Hepatic dysfunction has been associated with poor prognosis in critically ill patients. We aimed to investigate the incidence of early liver dysfunction and its association with probable predictive variables in a group of Iranian patients.

\section{METHODS}

The study was conducted on 149 pediatric patients referred to the pediatric intensive care unit (PICU), Shiraz University of Medical Sciences, Shiraz, Iran between April and October 2016 Serum levels of liver aminotransferase, alkaline phosphatase, total bilirubin, direct bilirubin, and international normalized ratio (INR) were recorded in 24, 48, and 96 hours after admission.

\section{RESULTS}

On the first day of admission, direct bilirubin was the least (9.1\%) and abnormal alkaline phosphatase level was the most $(66.9 \%)$ common abnormalities. Abnormal levels of all tests except alkaline phosphatase were predictive of increased rate of mortality. In univariable logistic regression, abnormal aminotransferases (ALT and AST), INR, total bilirubin, and direct bilirubin had significant relationship with patients' mortality after 24,48 , and 96 hours. In multivariable logistic regression only ALT and INR in the first 24 hours had significant relationship with mortality in final model. Although univariate logistic regression revealed a significant relationship between AST and ALT levels with PICU length of stay, no significant relationship was observed between these variables and PICU length of stay (except AST in the first 24 hours) in multivariable analysis.

\section{CONCLUSION}

Increase in liver enzymes may predict mortality and increased PICU length of stay in critically ill children.

\section{KEYWORDS:}

Intensive care units, Hepatic dysfunction, Mortality, Pediatric, Iran

Please cite this paper as:

Zahmatkeshan M, Serati Z, Freydooni S, Safarpour AR, Esmailnejad A, Haghbin S. Prediction of Early Liver Failure in Pediatric Patients Admitted to Intensive Care Unit. Middle East J Dig Dis 2019;11:141-146. doi: 10.15171/mejdd.2019.140.

\section{INTRODUCTION}

Hepatic dysfunction has been associated with poor prognosis in critically ill patients. Such patients are among the most carefully monitored patients in the hospital, controlled with many daily repeated tests. Interpretation of some of these tests included in the daily biochemical assessment can be challenging. ${ }^{1}$ Bilirubin and liver enzymes that constitute routine liver function test (LFT) panel demonstrate results in the abnormal range which may fluctuate during the admission period. It has been reported that early detection of some abnormalities, 
such as liver dysfunctions and their treatment can reduce mortality rates and improve health outcomes. ${ }^{2}$

Liver failure is a situation in which rapid deterioration of liver function results in altered mentation and coagulopathy in previously normal people. In such patients, disturbance in LFTs or overt hepatic dysfunction are apparent. Multiple mechanisms such as metabolic, hemodynamic, and inflammatory factors underline the pathophysiological pathways of liver damage. ${ }^{2,3}$ Laboratory measurement of liver function is available in most intensive care units (ICUs); therefore, determination of its clinical significance has both therapeutic and prognostic values. Indications such as disease severity and prognosis of patients must be used for selecting the patients requiring ICU care. Determining the prognosis of the patients is also useful for choosing the most effective treatments, which can lead to better clinical results. ${ }^{1,3}$ Based on the different markers that have been used as indicators of hepatic dysfunction, the prevalence of liver failure was reported to vary from $6 \%$ to $61 \%$ on the first day of admission in adult ICUs. ${ }^{2}$

Since bilirubin has been used as a key indicator of liver dysfunction in ICU critical illness scoring systems including Simplified "Sequential Organ Failure Assessment (SOFA)" score and "Simplified Acute Physiology Score (SAPS)", it has been the main focus of literature with regard to the liver failure. ${ }^{4}$ Previous studies have revealed that elevated serum bilirubin $>2 \mathrm{mg} / \mathrm{dL}$ was linked to the severity of illness and had a negative effect on both mortality and length of stay. ${ }^{4,5}$ As clinical jaundice develops only several days after hepatic injury ensues, liver dysfunction has been considered as a late event in sepsis and multi-organ failure. ${ }^{1}$ Moreover, it was seen that abnormal aspartate aminotransferase (AST), alkaline phosphatase (AKP), and gamma glutamyl transferase were correlated with increased risk of mortality within 30 days of admission in ICU. ${ }^{2}$

Therefore, early liver dysfunction is a subtle but prevalent phenomenon in adult critical illnesses and is associated with poor outcomes. However, there is a paucity of data evaluating LFTs on admission and through the subsequent days as a predictor of outcomes in critically ill children. In the present study we aimed to describe and clarify the prevalence, patterns, and significance of abnormal LFTs as a predictive marker of mortality and hospital length of admission of ill children in the absence of pre-existing liver diseases.

\section{MATERIALS AND METHODS}

\section{Ethics consideration}

All experimental procedures were approved by the Ethics Committee of Shiraz University of Medical Sciences, Shiraz, Iran and the study was in accordance with the Helsinki declaration of 1975, as revised in 2008 . Informed consent was obtained from the parents or guardians of the participants.

\section{Study subjects and setting}

This study was conducted prospectively in a 10-bed medical pediatric intensive care unit (PICU) at Nemazee Hospital affiliated to Shiraz University of Medical Sciences, Shiraz, Iran, between April and October 2013. The study group included 149 patients aged from 1 month to 18 years admitted to PICU. Patients with chronic liver diseases and hepatitis with viral, pharmacological, or toxic origin were excluded from the study. Children who died in the first 6 hours after admission and patients with readmission episodes were also excluded.

\section{Variables measurement}

The values of liver function tests including ALT, AST, AKP, total bilirubin (TBILI), direct bilirubin (DBILI), and international normalized ratio (INR) were recorded on the first day of admission as a part of a biochemical 'PICU profile'. All the tests were also repeated 48 and 96 hours after admission. In order to standardize the analysis, only the results of the tests taken in the morning rounds were recorded for the study. Information regarding demographic data, primary PICU diagnosis, PICU length of stay (LOS), Pediatric Risk of Mortality Score (PRISMIII) score, and outcomes were documented as well.

Liver enzymes level were considered abnormal if they were $\geq 1 / 4$ time of upper normal limit. ALT, TBILI, DBILI, and INR were also considered abnormal if they were higher than the upper limit of the laboratory levels. Because all the measurements were performed as a part of the routine metabolic evaluation of the patients and the confidentiality procedures were maintained, the requirement for informed consent was waived for the admission PICU profile, but written informed consents 
Table 1: Percentage of abnormal liver function tests results on 24, 48, and 96 hours after PICU admission

\begin{tabular}{|c|c|c|c|c|c|c|}
\hline & $\mathbf{A S T}^{1}$ & $\mathbf{A L T}^{2}$ & $\mathbf{A K} \mathbf{P}^{3}$ & TBILI $^{4}$ & DBILI $^{5}$ & INR $^{6}$ \\
\hline 24 hours after admission & 55.4 & 32.6 & 66.9 & 34.7 & 9.1 & 37.9 \\
\hline 48 hours after admission & 43.7 & 32.9 & 50.6 & 22.3 & 6.4 & 27.8 \\
\hline 96 hours after admission & 51.9 & 32.7 & 47 & 29.4 & 11.7 & 28 \\
\hline
\end{tabular}

${ }^{1}$ AST: Aspartate aminotransferase, ${ }^{2}$ ALT: Alanine aminotransferase, ${ }^{3}$ AKP: Alkaline phosphatase, ${ }^{4}$ TBILI: Total bilirubin, ${ }^{5}$ DBILI: Direct bilirubin, ${ }^{6}$ INR: International normalized ratio, PICU: Pediatric intensive care unit

Table 2: Univariable analysis of assessment of liver function tests on mortality rate at the first day of admission to PICU

\begin{tabular}{|c|c|c|c|c|c|}
\hline LFTs & & Alive (\%) & Dead $(\%)$ & $P$ value & Odds ratio \\
\hline \multirow{2}{*}{ AST } & $\mathrm{N}^{1}$ & 85 & 15.0 & \multirow{2}{*}{0.044} & \multirow{2}{*}{$\begin{array}{c}2.39 \\
(1-5.7)\end{array}$} \\
\hline & $\mathrm{AbNl}^{2}$ & 70.3 & 29.7 & & \\
\hline \multirow{2}{*}{ ALT } & $\mathrm{N}$ & 86 & 14 & \multirow{2}{*}{0.000} & \multirow{2}{*}{$\begin{array}{c}5.08 \\
(2.1-11.8)\end{array}$} \\
\hline & $\mathrm{AbN}$ & 54.8 & 45.2 & & \\
\hline \multirow{2}{*}{ AKP } & $\mathrm{N}$ & 75 & 25 & \multirow{2}{*}{0.85} & \multirow{2}{*}{-} \\
\hline & $\mathrm{AbN}$ & 76.4 & 23.6 & & \\
\hline \multirow{2}{*}{ TBILI } & $\mathrm{N}$ & 83.3 & 16.7 & \multirow{2}{*}{0.01} & \multirow{2}{*}{$\begin{array}{c}2.75 \\
(1.2-6.2)\end{array}$} \\
\hline & $\mathrm{AbN}$ & 64.4 & 35.6 & & \\
\hline \multirow{2}{*}{ DBILI } & $\mathrm{N}$ & 80.3 & 19.7 & \multirow{2}{*}{0.00} & \multirow{2}{*}{$\begin{array}{c}4.7 \\
(1.4-15.5)\end{array}$} \\
\hline & $\mathrm{AbN}$ & 46.2 & 53.8 & & \\
\hline \multirow{2}{*}{ INR } & $\mathrm{N}$ & 85 & 15 & \multirow{2}{*}{0.00} & \multirow{2}{*}{$\begin{array}{c}3.5 \\
(1.5-8.3)\end{array}$} \\
\hline & $\mathrm{AbN}$ & 61.2 & 38.8 & & \\
\hline
\end{tabular}

${ }^{1} \mathrm{~N}$ : Normal, ${ }^{2} \mathrm{AbN}$ : Abnormal, AST: Aspartate aminotransferase, ALT: Alanine aminotransferase, AKP: Alkaline phosphatase, TBILI: Total bilirubin, DBILI: Direct bilirubin,

INR: International normalized ratio, PICU: Pediatric intensive care unit, $p<0.05$ was considered as statistically significant

were obtained from the parents or guardians in case of repeated tests needed 48 and 96 hours after admission.

\section{Statistical analysis}

Data analyses were accomplished using SPSS software, version 19 (SPSS Inc., Chicago, IL, USA). Normal distribution of data was examined using KolmogorovSmirnov test. All quantitative variables were presented as mean \pm standard deviation of mean (SD) and qualitative variables were presented as frequency and percent. Independent student $\mathrm{t}$ test was used for comparison of quantitative variables. Paired t test, Chi-square, Fisher's exact test, and Mann-Whitney test were used as appropriate. To control confounders and find the variables related to dependent variables that fit the final predictive model, univariable and multivariable logistic regression analyses were performed. In all analyses, $p<0.05$ was considered as statistically significant.

\section{RESULTS}

A total of 149 patients admitted to PICU were en- rolled in this study. Among the participants, 76 (51\%) were male and $73(49 \%)$ were female and the patients' average age was 2 years (IQR: $0.58-8.4$ years). Mean PRISM III score was 6 (range 3 to 39) and overall mortality rate was $24.2 \%$ ( 36 out of 149). The most frequent causes of admissions were infectious diseases (22.1\%). Frequencies of neurological, respiratory, cardiac, and endocrine disorders were $19.4 \%, 15 \%, 11.4 \%$, and $10.7 \%$, respectively.

Abnormal serum AKP was the most frequent (66.9\%) and DBILI the least frequent abnormalities (9.1\%) detected on the first day of PICU admission. Assessment of LFTs 48 and 96 hours after admission also showed approximately the same frequency rates (table 1).

On the first day of PICU admission, abnormal levels of all tests except AKP revealed significant relationship with mortality. The results remained statistically significant even after adjusting for PRISM III score. This means that patients with normal test results were 2.39 times more likely to survive in PICU than those with abnormal test results (table 2). 
Table 3: Univariable analysis of assessment of liver function tests on mortality rate at 48 hours after admission to PICU

\begin{tabular}{|c|c|c|c|c|c|}
\hline LFTs & & Alive (\%) & Dead (\%) & $P$ value & Odds ratio \\
\hline \multirow{2}{*}{ AST } & $\mathrm{Nl}^{1}$ & 82.9 & 17.1 & \multirow{2}{*}{0.46} & \multirow{2}{*}{-} \\
\hline & $\mathrm{AbNl}^{2}$ & 75.0 & 2.0 & & \\
\hline \multirow{2}{*}{ ALT } & $\mathrm{N}$ & 87.5 & 12.5 & \multirow{2}{*}{0.04} & \multirow{2}{*}{$\begin{array}{c}3.5 \\
(1-11.6)\end{array}$} \\
\hline & $\mathrm{AbN}$ & 66.7 & 33.3 & & \\
\hline \multirow{2}{*}{$\mathrm{AKP}$} & $\mathrm{N}$ & 82.9 & 17.1 & \multirow{2}{*}{0.76} & \multirow{2}{*}{-} \\
\hline & $\mathrm{AbN}$ & 77.8 & 22.2 & & \\
\hline \multirow{2}{*}{ TBILI } & $\mathrm{N}$ & 85.5 & 14.5 & \multirow{2}{*}{0.01} & \multirow{2}{*}{$\begin{array}{c}4.4 \\
(1.1-6.1)\end{array}$} \\
\hline & $\mathrm{AbN}$ & 57.1 & 42.1 & & \\
\hline \multirow{2}{*}{ DBILI } & $\mathrm{N}$ & 83.1 & 16.9 & \multirow{2}{*}{0.00} & \multirow{2}{*}{$\begin{array}{c}22.4 \\
(2.2-22)\end{array}$} \\
\hline & $\mathrm{AbN}$ & 20.0 & 80.0 & & \\
\hline \multirow{2}{*}{ INR } & $\mathrm{N}$ & 83.3 & 16.7 & \multirow{2}{*}{0.00} & \multirow{2}{*}{$\begin{array}{c}5 \\
(1.5-15.2)\end{array}$} \\
\hline & $\mathrm{AbN}$ & 50.0 & 50 & & \\
\hline
\end{tabular}

${ }^{1} \mathrm{~N}$ : Normal, ${ }^{2} \mathrm{AbN}$ : Abnormal, AST: Aspartate aminotransferase, ALT: Alanine aminotransferase, AKP: Alkaline phosphatase, TBILI: Total bilirubin, DBILI: Direct bilirubin, INR: International normalized ratio, PICU: Pediatric intensive care unit, $p<0.05$ was considered as statistically significant

Table 4: Univariable analysis of assessment of liver function tests on mortality rate at 96 hours after admission to PICU

\begin{tabular}{|c|c|c|c|c|c|}
\hline LFTs & & Alive (\%) & Dead (\%) & $P$ value & Odds ratio \\
\hline \multirow{2}{*}{$\mathrm{AST}$} & $\mathrm{Nl}^{1}$ & 90.9 & 9.1 & \multirow{2}{*}{0.03} & \multirow{2}{*}{$\begin{array}{c}5.6 \\
(1-29.7)\end{array}$} \\
\hline & $\mathrm{AbNl}^{2}$ & 64.0 & 36.0 & & \\
\hline \multirow{2}{*}{ ALT } & $\mathrm{N}$ & 81.3 & 18.2 & \multirow{2}{*}{0.19} & \multirow{2}{*}{-} \\
\hline & $\mathrm{AbN}$ & 64.3 & 35.7 & & \\
\hline \multirow{2}{*}{$\mathrm{AKP}$} & $\mathrm{N}$ & 84.0 & 16.0 & \multirow{2}{*}{0.3} & \multirow{2}{*}{-} \\
\hline & $\mathrm{AbN}$ & 71.4 & 28.6 & & \\
\hline \multirow{2}{*}{ TBILI } & $\mathrm{N}$ & 84.8 & 15.2 & \multirow{2}{*}{0.02} & \multirow{2}{*}{$\begin{array}{c}4.8 \\
(1.1-20)\end{array}$} \\
\hline & $\mathrm{AbN}$ & 53.8 & 46.2 & & \\
\hline \multirow{2}{*}{ DBILI } & $\mathrm{N}$ & 80.5 & 19.5 & \multirow{2}{*}{0.04} & \multirow{2}{*}{$\begin{array}{c}6.1 \\
(0.88-43.4)\end{array}$} \\
\hline & $\mathrm{AbN}$ & 40.0 & 60.0 & & \\
\hline \multirow{2}{*}{ INR } & $\mathrm{N}$ & 84.4 & 15.6 & \multirow{2}{*}{0.00} & \multirow{2}{*}{$\begin{array}{c}12 \\
(2.6-55.8)\end{array}$} \\
\hline & $\mathrm{AbN}$ & 30.8 & 69.2 & & \\
\hline
\end{tabular}

${ }^{1} \mathrm{~N}$ : Normal, ${ }^{2} \mathrm{AbN}$ : Abnormal, AST: Aspartate aminotransferase, ALT: Alanine aminotransferase, AKP: Alkaline phosphatase, TBILI: Total bilirubin, DBILI: Direct bilirubin, INR: International normalized ratio, PICU: Pediatric intensive care unit, $p<0.05$ was considered as statistically significant

After 48 hours, the probability of death was still significantly higher in the groups with abnormal serum ALT, TBILI, DBILI, and INR levels (table 3 ) and after 96 hours, patients with abnormal TBILI, DBILI, and INR had a higher rate of mortality compared with those with normal test results. Moreover, in univariate analysis, patients with abnormal AST level were still 5.6 times more likely to die compared with those with normal results (OR: 5.6, 95\% CI: 1- 29.7, $P<0.03$ ) (table 4). In multivariable logistic regression, ALT and INR in the first 24 hours had significant relationship with mortality in final model (table 5).

Univariate logistic regression revealed that patients with abnormal AST and ALT had higher length of PICU stay (LOS) compared with the patients with normal aforementioned LFTs, at the first day of admission and 48 hours later $(p<0.05)$. Nevertheless, no significant differences were observed between the patients with normal and abnormal LFTs regarding the length of PICU stay at 48 and 96 hours after admission ( $p>0.05$, table 6). However, multivariable analysis showed no significant association between these variables and PICU length of stay, except for AST in the first 24 hours.

\section{DISCUSSION}

In this study, early liver dysfunction occurred within 24 hours of admission in $66.9 \%$ of critically ill children 
Table 5: Multivariable logistic regression analysis of assessment of liver function tests on mortality rate at 24 hours after admission in PICU

\begin{tabular}{lcccc}
\hline Variables & Constant & P value & OR & 95\% CI (U-L) \\
\hline AST & 1.76 & 0.001 & 0.17 & $0.68-0.43$ \\
\hline INR & 1.20 & 0.01 & 3.33 & $1.25-8.81$ \\
\hline
\end{tabular}

AST: Aspartate aminotransferase, INR: International normalized ratio

Table 6: Association between liver function tests and PICU length of stay

\begin{tabular}{|c|c|c|c|c|c|c|c|}
\hline & & \multicolumn{2}{|c|}{24 hours after admission } & \multicolumn{2}{|c|}{48 hours after admission } & \multicolumn{2}{|c|}{96 hours after admission } \\
\hline & & PICU LOS (Day) & P value & PICU LOS (Day) & P value & PICU LOS (Day) & P value \\
\hline \multirow{2}{*}{ AST } & $\mathrm{N}^{1}$ & $3.4 \pm 3.3$ & \multirow{2}{*}{0.00} & $4.3 \pm 5.3$ & \multirow{2}{*}{0.00} & $6.5 \pm 9.3$ & \multirow{2}{*}{0.8} \\
\hline & $\mathrm{AbNl}^{2}$ & $6.2 \pm 6.6$ & & $6.8 \pm 9.1$ & & $6 \pm 10.8$ & \\
\hline \multirow{2}{*}{ ALT } & $\mathrm{N}$ & $4.6 \pm 6.4$ & \multirow{2}{*}{0.01} & $5.5 \pm 6.1$ & \multirow{2}{*}{0.04} & $6.3 \pm 9.3$ & \multirow{2}{*}{0.09} \\
\hline & $\mathrm{AbN}$ & $6.9 \pm 6.3$ & & $6 \pm 8.9$ & & $5.5 \pm 11.8$ & \\
\hline \multirow{2}{*}{$\mathrm{AKP}$} & $\mathrm{N}$ & $5.5 \pm 5.8$ & \multirow{2}{*}{0.4} & $6.4 \pm 7.6$ & \multirow{2}{*}{0.7} & $6.4 \pm 10.2$ & \multirow{2}{*}{0.7} \\
\hline & $\mathrm{AbN}$ & $5.2 \pm 4.6$ & & $5.3 \pm 7.6$ & & $10.2 \pm 6$ & \\
\hline \multirow{2}{*}{ TBILI } & $\mathrm{N}$ & $5.1 \pm 5.1$ & \multirow{2}{*}{0.9} & $6.7 \pm 5.5$ & \multirow{2}{*}{0.1} & $6.1 \pm 9.6$ & \multirow{2}{*}{0.9} \\
\hline & $\mathrm{AbN}$ & $5.7 \pm 4.9$ & & $7.2 \pm 8.2$ & & $6.5 \pm 11.7$ & \\
\hline \multirow{2}{*}{ DBILI } & $\mathrm{N}$ & $5.5 \pm 4.9$ & \multirow{2}{*}{0.1} & $5.8 \pm 7.3$ & \multirow{2}{*}{0.1} & $6.4 \pm 10.3$ & \multirow{2}{*}{0.1} \\
\hline & $\mathrm{AbN}$ & $7.1 \pm 6.5$ & & $3.8 \pm 4.4$ & & $3.8 \pm 9$ & \\
\hline \multirow{2}{*}{ INR } & $\mathrm{N}$ & $5.5 \pm 5$ & \multirow{2}{*}{0.7} & $5.7 \pm 6.8$ & \multirow{2}{*}{0.8} & $6.3 \pm 9.2$ & \multirow{2}{*}{0.9} \\
\hline & $\mathrm{AbN}$ & $5.2 \pm 5$ & & $5.1 \pm 6.6$ & & $5.5 \pm 8.6$ & \\
\hline
\end{tabular}

${ }^{1} \mathrm{~N}$ : Normal, ${ }^{2} \mathrm{AbN}$ : Abnormal, AST: Aspartate aminotransferase, ALT: Alanine aminotransferase, AKP: Alkaline phosphatase, TBILI: Total bilirubin, DBILI: Direct bilirubin, INR: International normalized ratio, PICU: Pediatric intensive care unit, LOS: Length of stay, $p<0.05$ was considered as statistically significant,

without any previous history of liver problems and was an applicable predictor of PICU mortality. Both conditional logistic regression and logistic regression demonstrated independent effects of early hepatic dysfunction on mortality. It was also shown that increased level of liver enzymes (ALT and AST) could lead to patients' mortality after 24,48 , and 96 hours of admission and increased the PICU length of stay. Based on these results, liver dysfunction could be considered as a main independent prognostic factor for critically ill patients.

Results obtained in this research were in close agreement with a study that reported the frequency of $61 \%$ increased liver enzymes from medical and surgical adult ICU. ${ }^{2}$ However, different studies have reported hepatic dysfunction in $11-60 \%$ of cases based on the parameters they used. ${ }^{2,3,6}$ Hyperbilirubinemia, increased serum transaminases, alkaline phosphatase, lactate dehydrogenase, glutamyl-transferase, decreased serum albumin, and deranged coagulation are pivotal laboratory parameters for diagnosis of hepatic dysfunction. Although these parameters lack specificity and sensitivity, they emerge as a result of bile ducts or hepatocellular injuries and consequently widely used to predict the prognosis of patients in ICUs.

In a prospective multi-center cohort study, serum bilirubin $>2 \mathrm{mg} / \mathrm{dL}$ was used as an indicator of liver failure. Kramer and colleagues (2007) reported an independent link between liver dysfunction at the first 48 hours of ICU admission and mortality. ${ }^{3}$ TBILI has also been the main focus of such studies and has been used in ICU critical illness scoring systems, including Sequential Organ Failure Assessment and Simplified Acute Physiology Score. ${ }^{4,7}$ Kramer and co-workers (2007) disclosed that early hepatic dysfunction, which is defined as serum bilirubin $>2 \mathrm{mg} / \mathrm{dL}$ within 48 hours of admission, could be considered as a major prognostic factor in critically ill patients and increased in-hospital death. ${ }^{3}$ In another study conducted in 2010, $11 \%$ of the patients suffered from hypoxic hepatitis with prolonged prothrombin time had longer stay in ICU and higher mortality risk. ${ }^{8}$

In patients with trauma, hepatic derangement has been an independent predictor of increased mortality and ICU length of stay, irrespective of the absence or 
presence of renal dysfunction. ${ }^{9}$ It has been demonstrated that elevated ALT was associated with an increased risk of cardiovascular mortality in stable outpatient population. ${ }^{10}$ High peak ALT level was also identified as an independent predictor of worse clinical outcome and severe illness in patients with acute respiratory syndrome.

In agreement with our findings, Raurich and colleagues (2011) reported significant differences in serum levels of AST, LDH, INR, and creatinine between survivors and non-survivors, but not in their serum TBILI and ALT levels. ${ }^{11}$ Findings of the current study demonstrated that abnormal ALT and AST levels could lead to increased PICU length of stay. This was consistent with a previous study that proposed hepatic dysfunction as an independent predictor of mortality and increased ICU length of stay, irrespective of the absence or presence of additional organs dysfunction. ${ }^{9}$

Translating our results into clinical practice, ICU physicians should pay more attention to the systemic ischemic injuries in patients with elevated aminotransferase, TBILI, and DBILI, and deranged INR. These may lead to early recognition of hypo-perfusion and the implementation of specific treatments. In view of a great number of immunological, regulatory, and metabolic functions of liver, early liver dysfunction has a wide array of pathophysiological implications and should be considered as a main independent risk factor in critically patients. Determining the prognostic role of early hepatic dysfunction can prompt research into liver-protecting strategies, as these could play a critical role in controlling the mortality.

The present study had some limitations. We were unable to retrieve the necessary information after the patients were discharged from PICU. Therefore, the number of participating patients declined gradually 96 hours after admission and their data were not considered in statistical analysis. Identifying the exact cause of liver failure in all studied patients was also another limitation of this research.

\section{Source of Funding}

This research received no specific grant from any funding agency in public, commercial, or non-for-profit sectors.

\section{ACKNOWLEDGMENT}

Our thanks go to Hassan Khajehei PhD, for linguistic editing of the manuscript.
ETHICAL APPROVAL

There is nothing to be declared.

\section{CONFLICT OF INTEREST}

The authors declare no conflict of interest related to this work.

\section{REFERENCES}

1. Soultati A, Dourakis SP. Liver Dysfunction in the Intensive Care Unit. Ann Gastroenterol 2007;18:35-45.

2. Thomson SJ, Cowan ML, Johnston I, Musa S, GroundsM, Rahman TM. 'Liver function tests' on the intensive care unit: a prospective, observational study. Intensive Care Med 2009;35:1406-11. doi:10.1007/s00134-009-1511-7.

3. Kramer L, Jordan B, Druml W, Bauer P, Metnitz PG. Incidence and prognosis of early hepatic dysfunction in critically ill patients-a prospective multicenter study. Crit Care Med 2007;35:1099-104. doi:10.1097/01. CCM.0000259462.97164.A0.

4. Le Gall JR, Lemeshow S, Saulnier F. A new Simplified Acute Physiology Score (SAPS II) based on a European/North Americanmulticenter study.JAm Med Assoc 1993;270:295763. doi:10.1001/jama.1993.03510240069035.

5. Brienza N, Dalfino L, Cinnella G, Diele C, Bruno F, Fiore $\mathrm{T}$. Jaundice in critical illness: promoting factors of a concealed reality. Intensive Care Med 2006;32:267-74. doi: 10.1007/s00134-005-0023-3.

6. Durward A, Mayer A, Skellett S, Taylor D, Hanna S, Tibby SM, et al. Hypoalbuminaemia in critically ill children: incidence, prognosis, and influence on the anion gap. Arch Dis Child 2003;88:419-22. doi:10.1136/adc.88.5.419.

7. Vincent JL, Moreno R, Takala J, Willatts S, De Mendonça A, Bruining H, et al. The SOFA (Sepsis-related Organ Failure Assessment) score to describe organ dysfunction/ failure. On behalf of the Working Group on Sepsis-Related Problems of the European Society of Intensive Care Medicine. Intensive Care Med 1996;22:707-10.

8. Fuhrmann V, Jager B, Zubkova A, Drolz A. Hypoxic hepatitis - epidemiology, pathophysiology and clinical management. Wien Klin Wochenschr 2010;122:129-39. doi:10.1007/s00508-010-1357-6.

9. Harbrecht BG, Zenati MS, Doyle HR, McMichael J, Townsend RN, Clancy KD, et al. Hepatic dysfunction increases length of stay and risk of death after injury. $J$ Trauma 2002;53:517-23. doi:10.1097/01.TA.0000023161.76991.D1.

10. Ioannou GN, Weiss NS, Boyko EJ, Mozaffarian D, Lee SP. Elevated serum alanine aminotransferase activity and calculated risk of coronary heart disease in the United States. Hepatology 2006;43:1145-51. doi:10.1002/hep.21171.

11. Raurich JM, Llompart-Pou JA, Ferreruela M, Colomar A Molina M, Royo C,et al. Hypoxic hepatitis in critically ill patients: incidence, etiology and risk factors for mortality. J Anesth 2011;25:50-6. doi:10.1007/s00540-010-1058-3. 